\title{
Medical Intervention for Disease Stages Using Game Theory, Markov Chains, and Bayesian Inference
}

\author{
Ahmed Merie ${ }^{1}$, Myron Hlynka ${ }^{2}$ \\ ${ }^{1}$ School of Science, Florida Institute of Technology, Melbourne FL, USA \\ ${ }^{2}$ Dept. of Math \& Statistics, University of Windsor, Ontario, Canada \\ Correspondence: Ahmed Merie, School of Science, Florida Institute of Technology, Melbourne FL, USA. E-mail: \\ amerie2013@my.fit.edu
}

Received: February 5, 2019 Accepted: June 20, 2019 Online Published: June 27, 2019

doi:10.5539/ijsp.v8n4p60

URL: https://doi.org/10.5539/ijsp.v8n4p60

\begin{abstract}
In this paper, we study the progression of disease in the body, using Markov chains. We analyze the problem using game theory, and we use the results to estimate initial probabilities for our transition matrices. We also use Bayesian methods to obtain transition probabilities. We present three examples to explain how this process works.
\end{abstract}

Keywords: cancer, Markov chain, game theory, Bayesian inference

\section{Introduction and Literature Review}

In this paper, we study the progress of distribution of cancer in the body. There are many kinds of cancers. The most common include breast, bladder, colorectal, kidney, leukemia, lung, Non-Hodgkin's lymphoma, prostate. In our model, we assume that there are five levels of the disease (0,1,2,3 and 4). Zero here means there is no cancer.

We briefly discuss work that is relevant to our methodology in general and cancer in particular. Most previous work is quite different from our new view in this paper.

In section 2, we use a game theory model to estimate the distribution vector of the Markov chain. The players are the medication/treatment vs. the disease. The game theory model we create has a 5x5 matrix. The entries in the matrix represent the benefit/improvement of the body for the five different levels. The particular novelty of this paper is that it uses game theory to find the initial probability vector for the Markov chain introduced in section 3.

In section 3, we use a Markov chain to study the relationship between the cancer level now and after the next step. In other words, we create a probability transition matrix to understand the cancer process in the body. Our transition matrix is $5 \times 5$. The five states are (Stage 0 , stage I, ..., stage IV). We suggest two methods to estimate the transition matrix elements. We either use the most recent pair of medical tests, or we use Bayesian inference.

In section 4, We use Bayesian inference to estimate the probability transition values. We use conditional probability $P(A \mid B)$ when $X_{n+1}$ is $A$ and $X_{n}$ is $B$.

This paper studies how to model disease progression (cancer levels) for a patient over time.

In (Eichelsbacher: 1996), Eichelsbacher and Ganesh use Bayesian inference to estimate the transition probability matrix and the initial probability vector. They include no applications. We also consider estimates of the elements of the probability transition matrix using the same method (Bayesian approach), but we use a game theory model to estimate the initial probability vector, and we aim at a particular application.

In (Junsheng:2015), Junshege et al. used a continuous time Markov chain (CTMC) models to study the progression of chronic diseases in medical research. They assumed there are three states of the disease. They developed a Bayesian approach to evaluating the covariate effects on a (CTMC) model through a log-linear regression link. They simulate data and find that nutritional intervention is effective.

In (Hlynka:2008), Hlynka and Sajobi presented a class of "nice." $n \times n$ Markov probability transition matrices and infinitesimal generators whose limiting (steady state) probabilities are proportional to the first $n$ Fibonacci numbers. They used the limiting distribution vector to find the elements of the transition probability matrix. We can use such methods to generate a particular Markov chain transition matrix with a given limiting vector.

Articles $[1,3,4,6]$ provide additional details on the methods to be discussed. 


\section{Game Theory Model}

\subsection{Methodology}

To clarify further the nature of game theory in the disease model, we now contrast it with the theory of competitive equilibrium that is used between the first player (the treatment) and the second player (the disease). Treatments/medication include Surgery, Chemotherapy, Radiation Therapy, Immunotherapy, Medication, and any combination of these. Cancer growth can be local, or metastatic, slow or fast, and cancers try to avoid attack from the body's immune system. Every player attempts to obtain the best improvement by the competition against the other player.

We assume each player has five stages starting from 0 (which means the disease does not exist) to four (which means the disease is in the worst level). The orders of the stages concerning the disease present how much the disease dominates the body (or the percentage of the diseased cells in the body) and the stage of the drug is the level of the healthy part of the body. Consequently, it is not necessarily true that the stage of the disease is the complement of the stage of the medication because there are uncertainty levels in the model. In other words, the (medical) test result is not $100 \%$ right.

Our model is in Strategic Form. The goal of each player is getting more control of the body. This form is given by a triplet $(\mathbf{X}, \mathbf{Y}, \mathbf{A})$, where:

(1) $\mathbf{X}$ is a non-empty set, the set of strategies of Player 1 .

(2) $\mathbf{Y}$ is a non-empty set, the set of strategies of Player 2.

(3) $\mathbf{A}$ is a real-valued function defined on $\mathbf{X} \times \mathbf{Y}$. (Thus, $\mathbf{A}(\mathbf{X}, \mathbf{Y})$ is a real number for every $x \in \mathbf{X}$ and every $y \in \mathbf{Y}$ ).

The interpretation is as follows. During the test time, Player 1 is in stage $x \in \mathbf{X}$ and Player 2 is in stage $y \in \mathbf{Y}$, each unaware of the choice of the other. Then their choices are made known, and Player 1 wins $\mathbf{A}(\mathbf{X}, \mathbf{Y})$ from Player 2. Depending on the units we use, $\mathbf{A}(\mathbf{X}, \mathbf{Y})$ will be, for example, between $(-5,5)$. If $\mathbf{A}$ is negative, Player 1 lost against Player 2 by $\mathbf{A}$. Thus, $\mathbf{A}(\mathbf{X}, \mathbf{Y})$ represents the value for Player 1 (positive is optimistic and negative is pessimistic).

The model appears below:

Table 1. Payoff matrix

\begin{tabular}{|c|c|c|c|c|c|c|}
\cline { 3 - 7 } \multicolumn{2}{c|}{} & \multicolumn{6}{|c|}{ Disease } \\
\cline { 2 - 7 } \multicolumn{1}{c|}{$5 *$ Medication } & 0 & $a_{00}$ & $a_{01}$ & $a_{02}$ & $a_{03}$ & $a_{04}$ \\
\cline { 2 - 7 } & I & $a_{10}$ & $a_{11}$ & $a_{12}$ & $a_{13}$ & $a_{14}$ \\
\cline { 2 - 7 } & II & $a_{20}$ & $a_{21}$ & $a_{22}$ & $a_{23}$ & $a_{24}$ \\
\cline { 2 - 7 } & III & $a_{30}$ & $a_{31}$ & $a_{32}$ & $a_{33}$ & $a_{34}$ \\
\cline { 2 - 7 } & IV & $a_{40}$ & $a_{41}$ & $a_{42}$ & $a_{43}$ & $a_{44}$ \\
\hline
\end{tabular}

The first method we can use to find the optimal solution is to find the saddle points in the payoff matrix. A saddle point is a pair of strategies $(i, j)$ such that $a_{i m} \leq a_{i j}$, for all $m$, and $a_{m j} \leq a_{i j}$ for all $m$. The saddle points present optimal solutions. If there is no saddle point, we can solve the matrix above using linear programming for Player I (medication). We will find $P(0), P(I), P(I I), P(I I I), P(I V)$. These probabilities present the initial probabilities for the Markov Chain, as we will explain later. If there is a saddle point, the probability of the drug factor for that point is 1 , and the rest are zeros.

\subsection{Example 1}

In this example, we assume that there is a big difference between the test results and the real case of the patient. The diagonal elements of the matrix game are zeros. In other words, in game theory, when the test results are matching with the real case, the result is zero (No player win $a_{i j}=0$ at $i=j$ ). Under the same assumption, we full the rest of the table. For example, $a_{0,3}=-3$ means the player A (Medication) is losing three because we decide the patient is in a good situation follow the results test, but the disease is in the third level.

Below is the game theory matrix: 
Table 2. Example -1- payoff matrix

\begin{tabular}{|l||c|c|c|c|c|}
\hline Medication, Disease & 0 & I & II & III & IV \\
\hline \hline 0 & 0 & 1 & 2 & 3 & 4 \\
\hline I & 1 & 0 & 1 & 2 & 3 \\
\hline II & 2 & 1 & 0 & 1 & 2 \\
\hline III & 3 & 2 & 1 & 0 & 1 \\
\hline IIV & 4 & 3 & 2 & 1 & 0 \\
\hline
\end{tabular}

We use the "Vertex Enumeration Algorithm" in Python to solve this example (see Appendix A). The result is: $P(\Pi)=$ $(0,0,0,0,1)$

\subsection{Example 2}

In this example, we used a different assumption. In this case, as long as the result matching with the real case, the value of the game is zero, but if it's different, the value goes higher. For example, at $a_{I, I}$ the value is 0 because the result of the test is the same of the real case, while, $a_{I, I I}$ is 1 because the result is a little different than the real case. The table below shows all cases:

Table 3. Example -2- payoff matrix

\begin{tabular}{|l||c|c|c|c|c|}
\hline Medication, Disease & 0 & I & II & III & IV \\
\hline \hline 0 & 0 & 1 & 2 & 3 & 4 \\
\hline I & 1 & 0 & 1 & 2 & 3 \\
\hline II & 2 & 1 & 0 & 1 & 2 \\
\hline III & 3 & 2 & 1 & 0 & 1 \\
\hline IIV & 4 & 3 & 2 & 1 & 0 \\
\hline
\end{tabular}

The result of this example is: $P(\Pi)=(0.5,0,0,0,0.5)$

\subsection{Example 3}

Now, let takes another case. This time we made everything random. W

Table 4. example -3- payoff matrix

\begin{tabular}{|l||c|c|c|c|c|}
\hline Medication, Disease & 0 & I & II & III & IV \\
\hline \hline 0 & 0 & -1 & 2 & -2 & 2 \\
\hline I & -1 & 0 & -1 & 2 & -2 \\
\hline II & 2 & -1 & 0 & -1 & -2 \\
\hline III & -2 & 2 & -1 & 0 & -1 \\
\hline IIV & 2 & -2 & 2 & -1 & 0 \\
\hline
\end{tabular}

The result of this example is: $P(\Pi)=(0.11,0.26,0.25,0.26,0.12)$

\section{Markov Chain}

\subsection{Methodology}

A Markov chain is a collection of random variables $\Phi=\left\{\Phi_{n}: n \in T\right\}$, where $T$ is a countable time-set. It is customary to write $T$ as $\mathbb{Z}_{+}:=0,1, \ldots$, and we will do this henceforth.

Experimentally, the critical aspect of a Markov model, as opposed to any other set of random variables, is that it is forgetful of all but its most immediate past. The precise meaning of this requirement for the evolution of a Markov model in time is that the future of the process is independent of the past, given only its present value. For a process $\Phi$, evolving on a space $X$ and governed by an overall probability law $P$, to be a time-homogeneous Markov chain, there must be a set of "transition probabilities" $P_{n}(x, A), x \in X, A \subset X$ for appropriate sets $A$ such that for times $n, m \in \mathbb{Z}_{+}$. 
Definition 3.1 Let $P$ be a $k \times k$ matrix with elements $\left\{p_{i, j}: i, j=1, \ldots, k\right\}$. A random process $\left(X_{0}, X_{1}, \ldots\right)$ with finite state space $S=\left\{s_{1}, s_{2} \ldots, s_{k}\right\}$ is said to be a (homogeneous) Markov chain with transition matrix $P$, if for all $n$, all $i, j \in\{1, \ldots, k\}$ and all $i_{0}, \ldots, i_{n-1} \in\{1, \ldots, k\}$ we have $P\left(X_{n+1}=s_{j} \mid X_{0}=s_{i_{0}}, X_{1}=s_{i_{1}}, \ldots, X_{n ? 1}=s_{i_{(n-1)}}, X_{n}=s_{i}\right)=P\left(X_{n+1}=s_{j} \mid X_{n}=s_{i}\right)=$ $p_{i, j}$.

The probability $p_{i j}$ represents the probability that the process will make a transition to state $i$ given that currently the process is state $j$. Clearly one has

$$
p_{i j} \geq 0, \quad \sum_{i=0}^{\infty} p_{i j}=1, \quad j=0,1, . .
$$

Definition 3.2 The matrix containing the transition probabilities $P_{i j}$,

$$
P=\left[\begin{array}{ccc}
p_{00} & p_{01} & \cdots \\
p_{00} & p_{00} & \cdots \\
\vdots & \vdots & \ddots
\end{array}\right]
$$

is called the one-step probability transition matrix of the process.

Let us begin with a practical problem as motivation. In a cancer process, we assume there are five stages, namely $0, I, I I, I I I$ and $I V$. Research indicates that any stage of cancer may become higher, lower or stay in the same stage with a probability of $p_{i j}(\geq 0)$ when $(i<j, i<j$ or $i=j$ ) on the next step. The following is an important and exciting question. What will be the situation shared among the five levels of cancer in the long-run, or after a fixed number of steps? An important feature of this problem is that the future behavior of the disease depends on the current situation. Next, we formulate this problem by using a Markov chain model. For the five stages we assume for the cancer levels, we have a transition probability matrix $5 \times 5$

$$
P=\left[\begin{array}{ccccc}
p_{0,0} & p_{0, I} & p_{0, I I} & p_{0, I I I} & p_{0, I V} \\
p_{I, 0} & p_{I, I} & p_{I, I I} & p_{I, I I I} & p_{I, I V} \\
p_{I I, 0} & p_{I I, I} & p_{I I, I I} & p_{I I, I I I} & p_{I I, I V} \\
p_{I I I, 0} & p_{I I I, I} & p_{I I I, I I} & p_{I I I, I I I} & p_{I I I, I V} \\
p_{I V, 0} & p_{I V, I} & p_{I V, I I} & p_{I V, I I I} & p_{I V, I V}
\end{array}\right]
$$

In the probability transition matrix above $p_{i j}$ presents the probability of moving from stage $i$ to stage $j$. If we assume this matrix is like a random walk matrix, that means $p_{i j}=0$ for all $i>j+1$ or $i<j-1$. Our model is note quite a random walk model, but we still think the probability values when $i>j+1$ or $i<j-1$ are very small because, for instance, it is very rare for the patient to move from stage I to stage III or IV in one step (but it is not impossible so it is not zero).

As we know that, for any irreducible and aperiodic Markov chain having $k$ states, there exists one stationary distribution, and for any initial distribution $\Pi^{(0)}$.

$$
\lim _{n \rightarrow \infty} P^{(n)} \Pi^{(0)}=\Pi
$$

Where $\Pi$ is the unique stationary distribution vector for the transition matrix $P$. If we need to know the progression of the disease after six test results, for example, we can find the sixth step probability transition matrix without calculating the initial distribution. In this case, we don't need the game theory model at all. But if we want $\Pi^{(6)}$, then we do need $\Pi^{(0)}$, which is where our game theory result can be useful.

As we see, we need to estimate the initial distribution victor $\Pi^{(0)}$ and the one-step probability transition matrix of the process. We think the best estimation vector for the initial distribution is the result of our Game theory model. As for the one-step probability transition matrix, there are two suggestions. The first one is to use the most recent two medical tests for the patient and to estimate the conditional probability from them. The advantage of this method is that we use the same patient to estimate the likelihood of his/her change. The disadvantage is we need to wait until we get two tests which takes time, and this is not good for the patient's health. The second method is using Bayesian inference.

\subsection{Example 1}

In this example, we use the same assumption as in example 1 of Section 2 . The suggested probability transition matrix is: 


\begin{tabular}{|l||c|c|c|c|c|}
\hline Medication, Disease & 0 & I & II & III & IV \\
\hline \hline 0 & 0 & -1 & 2 & -2 & 2 \\
\hline I & -1 & 0 & -1 & 2 & -2 \\
\hline II & 2 & -1 & 0 & -1 & -2 \\
\hline III & -2 & 2 & -1 & 0 & -1 \\
\hline IIV & 2 & -2 & 2 & -1 & 0 \\
\hline
\end{tabular}

Table 5. Example -1- PTM

The two step PTM is

$$
P=\left[\begin{array}{lllll}
0.25 & 0.34 & 0.22 & 0.16 & 0.03 \\
0.20 & 0.34 & 0.24 & 0.18 & 0.04 \\
0.16 & 0.32 & 0.24 & 0.20 & 0.08 \\
0.14 & 0.32 & 0.24 & 0.20 & 0.10 \\
0.07 & 0.22 & 0.26 & 0.24 & 0.21
\end{array}\right]
$$

The PTM after $\mathrm{n}$ steps, for large $\mathrm{n}$, is

$$
P=\left[\begin{array}{lllll}
0.179 & 0.323 & 0.238 & 0.189 & 0.071 \\
0.179 & 0.323 & 0.238 & 0.189 & 0.071 \\
0.179 & 0.323 & 0.238 & 0.189 & 0.071 \\
0.179 & 0.323 & 0.238 & 0.189 & 0.071 \\
0.179 & 0.323 & 0.238 & 0.189 & 0.071
\end{array}\right]
$$

This output means without any initial probability values, the eventual probability of the future cases of the patients are $P(0)=0.179, P(I)=0.323, P(I I)=0.238, P(I I I)=0.189$ and $P(I V)=0.71$. The highest probability is for case $I I$. However, the main strength of our game theory approach is that it applies more appropriately to smaller $\mathrm{n}$ step situations.

If we use the initial probability we got from example 1 of the game theory we got $P(0)=0.00, P(I)=0.00, P(I I)=$ $0.10, P(I I I)=0.10$ and $P(I V)=0.40$. For example 2 we got $P(0)=0.20, P(I)=0.10, P(I I)=0.10, P(I I I)=0.10$ and $P(I V)=0.20$. But using the third example we got $P(0)=0.198, P(I)=0.228, P(I I)=0.228, P(I I I)=0.226$ and $P(I V)=0.198$. Another possible transition matrix could choose to have one state represent a complete cure and another state represent death. In this case, we would have a Markov chain with two absorbing states. The computation for a small number of steps would be the same as just illustrated.

\section{Bayesian Inference}

Bayesian probability differs from classical probability. In Bayesian thinking, probabilities are treated as an expression of belief together with prior information. Therefore it is perfectly reasonable for a Bayesian to say "I am 70\% sure that this patient has stage II cancer". By combining prior beliefs, and current events (the first test result), one can compute the posterior, i.e., the probability that the cancer is in stage II. The idea behind Bayesian thinking is to keep updating the beliefs as more evidence is provided. Since this approach deals with confidence, it is usually referred to as a personal view on probability. Bayesian inference is closely related to the Bayesian perspective on probability, in the sense that it manipulates priors, evidence, and likelihood to compute the posterior.

A sequence $X_{0}, X_{1}, \ldots$ of random variables on a common probability space $(\Omega, F(\Omega), P)$ is referred to a discrete time parameter stochastic process. Let $\left\{X_{n}\right\}$ be a Markov chain on $\Omega$ with the unknown probability transition matrix $P$. If we assume $p_{i j} i, j=0, I, I I, I I, I V$, is a Bayesian procedure based on the observations $X_{0}, X_{1}, \ldots, X_{n+1}$

$$
p_{i, j}=\frac{\sum_{k=1}^{n} \mathbf{I}\left(X_{k}=i, X_{k+1}=j\right)}{\sum_{k=1}^{n} \mathbf{I}\left(X_{k}=i\right)},
$$

whetre $I()$ is an indicator function. Now the conditional probabilities of the transition probability matrix are ready. Also, we have the initial probability from game theory. Our model is ready to apply. 


\section{Discussion}

In the first part of this study, we used the Game Theory to estimate the initial distribution vector for three different assumptions (1- if the difference between the real stage and the test results is big, 2- it is neither big nor small, 3- it is small). We found three different victors, as shown in examples 1,2 and 3. In Section 3, we used Markov Chains to understand how the cancer cells can fight chemotherapy or any other treatments during the treatment periods. We also considered three different cases. Then in the same section, we used the Transition Matrix to observe the stage of the disease with and without using the initial probability vector. In Section 4, we suggested the use of Bayesian inference to create the transition matrix depending on our observations from the previous medical treatments.

\section{References}

Becque, T., White, I., \& Haggard, M. (2015). A causal model for longitudinal randomised trials with time-dependent non-compliance. Statistics in Medicine, 34, 2019-2034, Wiley. https://doi.org/10.1002/sim.6468

Eichelsbacher, P., \& Ayalvadi, G. (2002). Bayesian Inference for Markov Chains. Journal of Applied Probability, 39(1), 91C99. https://doi.org/10.1239/jap/1019737990

Erguler, K., Chandra, N. L., Proestos, Y., Lelieveld, J., Christophides, G. K., \& Parham, P. E. (2017). A large-scale stochastic spatiotemporal model for Aedes albopictus-borne chikungunya epidemiology. PLoS ONE , 12(3). https://doi.org/10.1371/journal.pone.0174293

Gamerman, D., \& Lopes, H. F. (2006). Markov chain Monte Carlo : Stochastic Simulation for Bayesian Inference. Chapman \& Hall/CRC 2nd.

Hlynka, M., \& Sajobi, T. F. (2008). A Markov Chain Fibonacci Model. Missouri Journal of Mathematical Sciences, 20(3), 186-199.

Lunn, D., Barrett, J., Sweeting, M., \& Thompson, S. (2013). Fully Bayesian hierarchical modelling in two stages with application to meta-analysis, Appl. Statist., 62(4), 551-572.

Ma, J., Chan, W., Tsai, C., Xiong, M., \& Tilley, B. (2015). Analysis of trans theoretical model of health behavioral changes in a nutrition intervention studyła continuous time Markov chain model with Bayesian approach, Statistics in Medicine, 34, 3577-3589, Wiley.

\section{Appendix A Examples' Solution of Game Theory}

\section{Example 1,2,3}

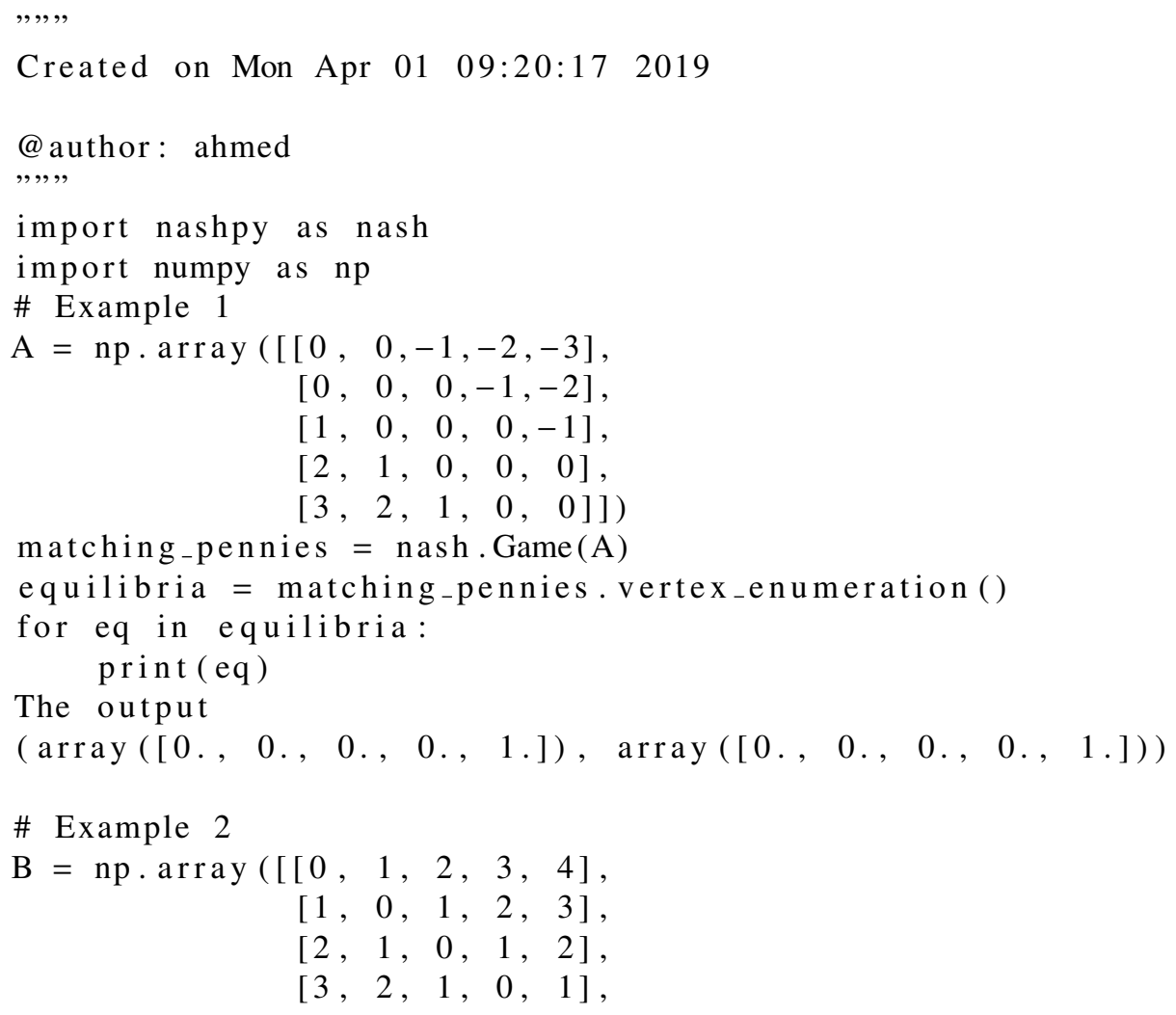


$[4,3,2,1,0]])$

matching-pennies = nash.Game(B)

equilibria = matching_pennies.vertex_enumeration()

for eq in equilibria:

print (eq)

$\left(\operatorname{array}([0.5,0 ., 0 ., 0 ., 0.5]), \operatorname{array}\left(\left[\begin{array}{lll}0.5,0 ., 0 ., 0 ., 0.5 & 0 .)\end{array}\right)\right.\right.$

\# Example 3

$\mathrm{C}=\mathrm{np}$. array $([[0,-1,2,-2,2]$,

$[-1,0,-1,2,-2]$,

$[2,-1,0,-1,2]$,

$[-2,2,-1,0,-1]$,

$[2,-2,2,-1,0]])$

matching pennies $=$ nash. Game $(\mathrm{C})$

equilibria $=$ matching_pennies. vertex_enumeration( $)$

for eq in equilibria:

print (eq)

( array ([0.1147541, $0.26229508,0.24590164,0.26229508,0.1147541])$,

array $([0.1147541,0.26229508,0.24590164,0.26229508,0.1147541]))$

\section{Appendix B Example' Solution of TPM}

$, ", \cdots$

Created on Mon Apr 08 11:20:17 2019

@ author: ahmed

,,,,

import numpy as np

from numpy. linalg import matrix - power

$\mathrm{A}=\mathrm{np}$. array $\left(\left[\begin{array}{lllll}0.4, & 0.3, & 0.2,0.1,0.0\end{array}\right]\right.$,

$\left[\begin{array}{lllll}0.2 & 0.4,0.2,0.2,0.0\end{array}\right]$

$[0.1,0.4,0.2,0.2,0.1]$

$[0.1,0.2,0.4,0.2,0.1]$,

$[0.0,0.1,0.2,0.3,0.4]])$

$\mathrm{a}=\mathrm{np}$. array $([0.00,0.00,0.00,0.00,1.00])$

$\mathrm{b}=\mathrm{np}$. array $([0.50,0.00,0.00,0.00,0.50])$

$\mathrm{c}=\mathrm{np}$. array $([0.11,0.26,0.25,0.26,0.11])$

matrix_power (A, 2)

print (" ")

print ("The second Power of the Matrix")

print ("============================-")

print ( matrix_power (A, 2))

print (" ")

print ("The 20 th Power of the Matrix")

print ("==============================”)

print (np . around (matrix_power $(\mathrm{A}, 20), 3))$

print (" ")

print ("Long-Run Behavior of Markov Chains Using EX1 of Game Theory")

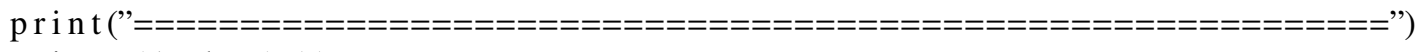

print (A.dot (a))

print (" ")

print ("Long-Run Behavior of Markov Chains Using EX2 of Game Theory")

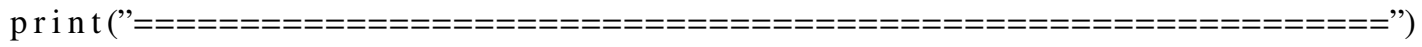

print (A.dot (b))

print (" ") 


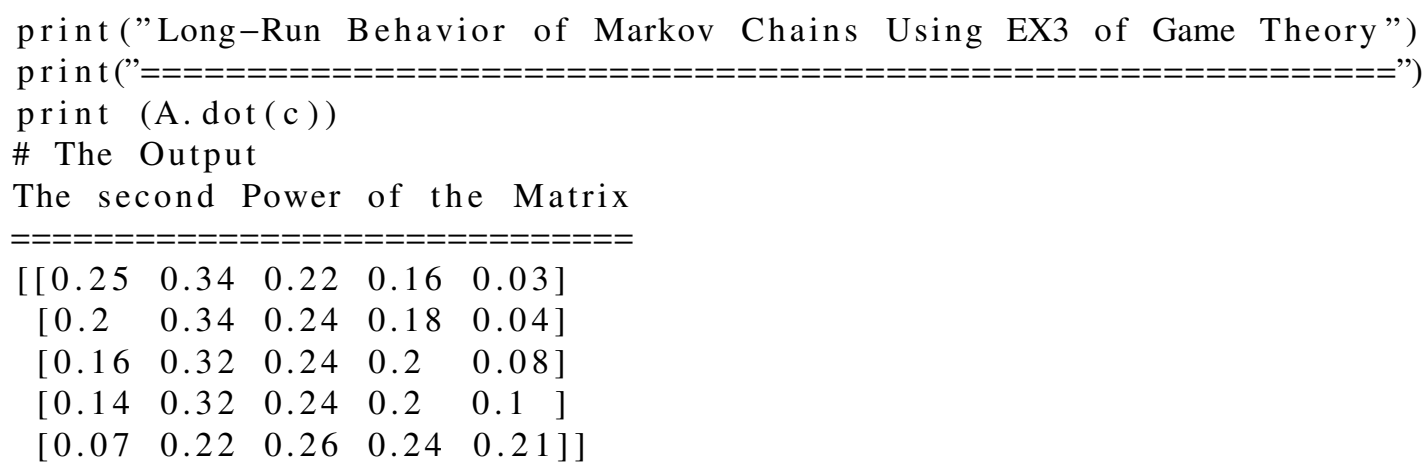

The 20 th Power of the Matrix

\begin{tabular}{|c|c|c|c|c|}
\hline$[[0.179$ & 0.323 & 0.238 & 0.189 & $0.071]$ \\
\hline$[0.179$ & 0.323 & 0.238 & 0.189 & $0.071]$ \\
\hline$[0.179$ & 0.323 & 0.238 & 0.189 & $0.071]$ \\
\hline$[0.179$ & 0.323 & 0.238 & 0.189 & $0.071]$ \\
\hline$[0.179$ & 0.323 & 0.238 & 0.189 & $0.071]$ \\
\hline
\end{tabular}

Long-Run Behavior of Markov Chains Using EX1 of Game Theory

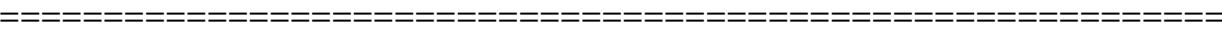
$\left[\begin{array}{lllll}0 . & 0 . & 0.1 & 0.1 & 0.4\end{array}\right]$

Long-Run Behavior of Markov Chains Using EX2 of Game Theory

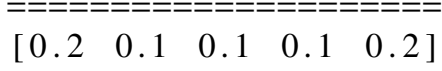

Long-Run Behavior of Markov Chains Using EX3 of Game Theory

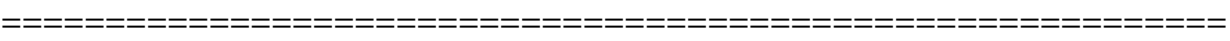
$\left[\begin{array}{lllll}0.198 & 0.228 & 0.228 & 0.226 & 0.198\end{array}\right]$

\section{Copyrights}

Copyright for this article is retained by the author(s), with first publication rights granted to the journal.

This is an open-access article distributed under the terms and conditions of the Creative Commons Attribution license (http://creativecommons.org/licenses/by/4.0/). 